Arq. Bras. Med. Vet. Zootec., v.70, n.1, p.117-121, 2018

\title{
Estudo comparativo entre métodos manual e digital no cálculo do ângulo do platô tibial em cães
}

\author{
[Comparative study of manual and digital method in the calculation of the tibial plateau \\ angle in dogs] \\ A.M. Alves ${ }^{1}$, S. Capelasso ${ }^{1}$, R. Zanatta ${ }^{1}$, R.S.L. Oliveira ${ }^{1}$, M.D. Santos ${ }^{1}$, F.M. Silva ${ }^{1}$, \\ C.R.A. Ferrigno ${ }^{2}$, N.M.B. Dower ${ }^{3}$, S.H. Freitas ${ }^{1}$, K.C.I. Yamauchi ${ }^{1 *}$ \\ ${ }^{1}$ Faculdade de Medicina Veterinária - UNIC - Cuiabá, MT \\ ${ }^{2}$ Faculdade de Medicina Veterinária e Zootecnia - USP - São Paulo, SP \\ ${ }^{3}$ Faculdade de Medicina Veterinária - UFMT - Cuiabá, MT \\ RESUMO
}

\begin{abstract}
São poucos os dados publicados sobre a aplicabilidade ou a confiabilidade dos métodos digitais para mensuração do ângulo do platô tibial (APT) em comparação à mensuração manual. $\mathrm{O}$ objetivo deste estudo foi comparar o APT obtido pelo cálculo manual padrão, em filmes radiográficos, com os valores obtidos por cálculos em imagens digitais, por meio do software do computador. Foram analisadas radiografias digitais do membro pélvico, direito e esquerdo, em perfil verdadeiro, de 20 cães saudáveis, com idade superior a um ano. Três observadores com graus de experiência semelhantes em cálculo do APT realizaram as mensurações em cada radiografia. Primeiramente, foram calculados os ângulos dos platôs tibiais manualmente nas películas radiográficas e, posteriormente, os cálculos foram realizados na imagem digital, por meio do software para medicina veterinária do sistema de raios X digital. Os resultados do presente estudo demonstraram diferenças significativas entre os métodos, com valor das médias do cálculo digital $20,48 \pm 3.71$ e do cálculo manual $23.90 \pm 4.02$. Valor de $\mathrm{P}$ foi $<0,01$ na comparação entre médias dos dois grupos. Na avaliação interobservadores, houve diferença significativa no cálculo manual no perfil direito, o que sugere maior variação no cálculo manual quando comparado ao digital. O cálculo realizado pelo software apresentou menor variação nos valores interobservadores. Acredita-se que a menor variação obtida nos resultados aqui apresentados se dê em razão das facilidades proporcionadas pelo software. Conclui-se que houve diferença entre os valores obtidos por meio dos métodos convencional e digital no cálculo do ângulo do platô tibial, sendo os valores digitais menores entre os observadores, e que o método digital no cálculo do ângulo do platô tibial levou à menor variação quando comparado ao método manual.
\end{abstract}

Palavras-chave: ligamento cruzado cranial, mensuração, software, ruptura

\begin{abstract}
There are few published data on the applicability and reliability of digital methods for measuring the TPA compared with manual measurement. The objective of this study was to compare the TPA obtained by calculating standard manual on conventional radiographs, with the values obtained by calculations in digital images through a computer program. Bilateral digital radiographs of hind limb were analyzed in true profile for 20 healthy dogs older than two years. Three observers with similar levels of experience in calculating the TPA conducted measurements on each radiograph. First we calculated the angle of the tibial plateaus manually in $x$-ray film and then the calculations were made through the program offered by the computer program in the digital image. The results of this study showed significant differences between the methods, with average value of 20.48 \pm 3.71 digital calculating average and $23.90 \pm 2.4$ in the manual calculation. $P$ value was $<0.01$ when comparing averages. No significant difference in manual calculation in right profile were found between interobserver assessments, suggesting greater variation
\end{abstract}

Recebido em14 de julho de 2016

Aceito em 3 de abril de 2017

E-mail: itokelly@yahoo.com.br 
in manual calculation compared to digital. The calculation performed by the software showed less variation in values. The slight variation obtained in our results is believed to be due to the facilities provided by the software. There was a difference between the values obtained by means of conventional and digital methods for calculating the angle of the tibial plateau, and the smaller digital values between the observers and the digital method in the calculation of the tibial plateau angle led to less variation when compared to the manual method.

Keywords: cranial cruciate ligament, measurement, program, rupture

\section{INTRODUÇÃO}

A mensuração precisa do ângulo do platô tibial (APT) é fundamental para avaliação e planejamento de cirurgias de ruptura de ligamento cruzado cranial em cães (Buquera et al., 2004; Harasen, 2002). Múltiplos fatores podem estar potencialmente associados a essa afecção, sendo o ângulo do platô tibial um deles (Johnson J. e Johnson A., 1993; Lazar et al., 2005; Griffon, 2010). Todas essas mensurações antes da era digital eram realizadas em radiografias obtidas por meio de técnicas convencionais manuais (Morris e Lipowitz, 2001; Wilke et al., 2002; Fettig et al., 2003). Com as novas tecnologias, métodos mais avançados, como a radiografia digital, tornaramse padrão em muitos hospitais veterinários. Como resultado, tem havido um aumento na utilização de métodos digitais para mensuração do APT em cães (Reif et al., 2004; Serwa et al., 2009).

São poucos os dados publicados sobre a aplicabilidade ou a confiabilidade dos métodos digitais para mensuração do APT (Reif et al., 2004; Serwa et al., 2009). Acredita-se que as diferenças nas etapas de mensuração de cada método, pequenos detalhes, como diâmetro da ponta da caneta, habilidade no uso do transferidor e habilidade no uso do software de medição, podem potencialmente resultar em diferenças entre as medidas do APT obtido por métodos convencionais e digitais.

Por conseguinte, o objetivo do presente estudo foi comparar os métodos manual e digital de avaliação do APT por meio de variação nos valores do APT.

\section{MATERIAL E MÉTODOS}

O trabalho foi submetido à Comissão de Ética de Uso de Animais da Universidade de Cuiabá e aprovado, protocolado sob o número 012/2014. As imagens radiográficas foram realizadas e avaliadas no Setor de Radiologia do Hospital Veterinário da Universidade de Cuiabá (Unic). Os proprietários de cães incluídos no estudo assinaram um termo de consentimento, por meio do qual permitiam que toda a documentação sobre seus cães pudesse ser utilizada para pesquisa científica e publicação. Foram utilizados 20 cães, entre um ano e seis meses até sete anos, saudáveis, sem predileção de sexo e raças variadas.

As imagens digitais em perfil verdadeiro de ambas as tíbias foram obtidas utilizando-se o aparelho de raios $\mathrm{X}$ marca SAWAE, modelo Altus ST503-HF, e o sistema Carestream CR Vita Veterinário, com software Carestream Image Suite. Os filmes radiográficos, utilizados para o cálculo manual, foram obtidos por meio da impressão das mesmas imagens digitais, sem manipulações, utilizando-se impressora laser DryView 5700.

Todas as imagens radiográficas incluíram a tíbia, o tarso, o fêmur distal e foram centradas na articulação do joelho com superposição dos côndilos femorais e tibiais. As imagens calculadas manualmente foram provenientes da mesma unidade processadora automática para radiografia digital, sem manipulações posteriores.

As imagens radiográficas da tíbia direita e da esquerda foram avaliadas por três observadores, médicos veterinários, com experiência em cálculo do ângulo do platô tibial. A ordem das radiografias foi aleatória entre as medições. Para cada imagem, foram feitos o cálculo manual e o digital pelos três avaliadores, individualmente.

Para o cálculo do platô tibial no filme radiográfico, o método utilizado foi o descrito por Slocum e Devine (1983). Foram fornecidos caneta permanente, régua de plástico transparente de 30 centímetros e um transferidor 
de plástico transparente idênticos para cada avaliador.

O software veterinário possui uma ferramenta de mensuração de osteotomia de nivelamento do platô tibial para cálculo do APT. Com fácil manuseio operacional, o aplicativo solicita marcação de uma linha unindo os pontos anterior e posterior do platô tibial, bem como de uma linha no eixo mecânico da tíbia traçado das eminências intercondíleas ao centro do osso tarso. Automaticamente o software fornece o valor do ângulo do platô tibial.

A análise estatística dos dados foi resumida como média e ANOVA, pelo teste de Duncan; o método foi utilizado para avaliar a correlação entre os valores obtidos por métodos convencionais e digitais. O emparelhamento do teste ANOVA, seguido por teste de Duncan, foi utilizado para comparar os valores obtidos por cálculo convencional manual com os valores obtidos por meio de cálculos digitais no software. Todos os resultados foram avaliados com os valores de $\mathrm{P} \leq 0,05$, considerados significativos.

\section{RESULTADOS}

Cada animal forneceu 20 imagens em perfil verdadeiro do membro esquerdo e 20 imagens do membro direito, totalizando 40 imagens. Cada avaliador calculou o ângulo do platô tibial 80 vezes em 40 imagens, sendo 40 calculadas de maneira convencional ( 20 no perfil direito e 20 no esquerdo) e 40 (20 no perfil direito e 20 no esquerdo) calculadas por meio do programa do aparelho digital.

As considerações dos avaliadores sobre os métodos foi que o cálculo por meio do software é mais rápido, fácil e prático quando comparado com o método convencional.

Na comparação do perfil direito e do esquerdo, separadamente, em função do método manual ou do software com 60 dados (20 valores de cada avaliador no perfil direito e 20 valores no perfil esquerdo), foi demonstrada diferença significativa entre os métodos, sendo os valores obtidos por meio do programa digital menores nos dois grupos (no perfil direito e no esquerdo) (Tab. 1).

Quando foram avaliados os 120 valores dos ângulos (perfil direito e perfil esquerdo de todos os observadores) na comparação entre os métodos manual e software digital, também foi observada diferença estatística, sendo menores os valores obtidos por meio do programa digital (Tab. 2).

Foram comparados os valores dos ângulos do platô do perfil direito e do esquerdo, obtidos por meio do cálculo manual e do programa digital. Não foi encontrada diferença significativa nos valores dos ângulos calculados no perfil direito e no esquerdo, independentemente do método utilizado (Tab. 3).

Tabela 1. Valores de médias com desvio padrão das imagens em perfil dos membros pélvicos direito e esquerdo avaliados pelos três observadores, por meio dos programas digital e convencional, demonstrando diferença significativa entre os valores obtidos pelos dois métodos, sendo os valores obtidos pelo programa digital menores nos dois perfis

\begin{tabular}{cccc}
\hline Método & $\begin{array}{c}\text { Número imagens } \\
\text { avaliadas }\end{array}$ & Perfil direito & Perfil esquerdo \\
\hline Software & 60 & $20.10 \pm 3.96^{\mathrm{A}}$ & $20.86 \pm 3.44^{\mathrm{A}}$ \\
Manual & 60 & $23.73 \pm 4.28^{\mathrm{B}}$ & $24.08 \pm 3.78^{\mathrm{B}}$ \\
\hline${ }^{\mathrm{A}, \mathrm{B}}$ Letras diferentes das médias do ângulo na mesma coluna diferem entre si, $\mathrm{P} \leq 0,01$, pelo teste $\mathrm{F}$.
\end{tabular}

Tabela 2. Valores de médias com desvio padrão de todas as imagens obtidas, somando-se as imagens em perfil do membro pélvico direito e do esquerdo avaliados pelos três observadores, por meio dos programas digital e convencional, demonstrando diferença significativa entre os valores obtidos pelos dois métodos, sendo menor o valor obtido pelo programa digital

\begin{tabular}{ccc} 
Método & Número imagens avaliadas & Perfil direito e perfil esquerdo \\
\hline Software & 120 & $20,48 \pm 3.71^{\mathrm{A}}$ \\
Manual & 120 & $23.90 \pm 4.02^{\mathrm{B}}$ \\
\hline
\end{tabular}

\footnotetext{
${ }^{\mathrm{A}, \mathrm{B}}$ Letras diferentes das médias do ângulo na mesma coluna diferem entre si, $\mathrm{P} \leq 0,01$, pelo teste $\mathrm{F}$.
} 
Alves et al.

Tabela 3. Valores de médias com desvio padrão comparando o perfil direito e o esquerdo para os métodos do cálculo manual e do software, demonstrando que não houve diferença estatística

\begin{tabular}{cccc|}
\hline & Número de imagens avaliadas & Manual & Software \\
\hline Perfil direito & 60 & $23,73 \pm 4.28$ & $20,10 \pm 3.96$ \\
Perfil esquerdo & 60 & $24.08 \pm 3.78$ & $20,86 \pm 3.44$ \\
Valor de P & & 0,22 & 0,26 \\
\hline
\end{tabular}

\section{DISCUSSÃO}

Este estudo abrange tema de aplicação prática na rotina hospitalar e orienta os profissionais da área quanto ao método mais preciso do cálculo do ângulo do platô tibial e ao planejamento préoperatório de cirurgias de ruptura de ligamento cruzado cranial. Serwa et al. (2009) realizaram estudo semelhante, cujo resultado não apresentou diferença significativa entre os métodos, validando, assim, o método digital que, até então, não era confiável, quando comparado ao método convencional, e concluíram, também, que o software é um método fácil e rápido de ser realizado. Este trabalho demonstrou resultado diferente de Serwa et al. (2009), quando comparados os métodos manual e digital, entretanto corrobora que o método digital para as medidas do APT foi mais fácil e mais rápido de ser realizado do que o método convencional, o que demonstra superioridade do método digital para determinar o valor do APT.

Foram comparadas avaliações por observador em relação ao método utilizado e houve diferença significativa somente no cálculo manual no perfil direito, o que sugere maior variação no cálculo manual do que no programa digital (Tab. 4 e 5).

Tabela 4. Média dos valores calculados por meio do método manual, por observador, nos perfis direito e esquerdo, demonstrado diferença estatística entre os avaliadores no perfil direito

\begin{tabular}{cccc}
\hline Avaliador & Imagens avaliadas & $\begin{array}{c}\text { Perfil direito } \\
\text { (Manual) }\end{array}$ & $\begin{array}{c}\text { Perfil esquerdo } \\
\text { (Manual) }\end{array}$ \\
\hline Avaliador 1 & 20 & $22.65 \pm 5.08^{\mathrm{B}}$ & $24.80 \pm 4.42$ \\
Avaliador 2 & 20 & $25.65 \pm 3.16^{\mathrm{A}}$ & $24.60 \pm 3.64$ \\
Avaliador 3 & 20 & $22.90 \pm 3.90^{\mathrm{B}}$ & $22.85 \pm 3.04$ \\
Valor de P & & 0,04 & 0,2 \\
\hline${ }^{\mathrm{A}, \mathrm{B}}$ Letras diferentes das médias do ângulo na mesma coluna diferem entre si, P $\leq 0,05$, pelo teste $\mathrm{F}$.
\end{tabular}

Tabela 5. Média dos valores calculados por meio do software, por observador, nos perfis direito e esquerdo, demonstrado que não há diferença estatística entre os avaliadores no perfil direito

\begin{tabular}{cccc} 
Avaliador & $\begin{array}{c}\text { Imagens } \\
\text { avaliadas }\end{array}$ & $\begin{array}{c}\text { Perfil direito } \\
\text { (Software) }\end{array}$ & $\begin{array}{c}\text { Perfil esquerdo } \\
\text { (Software) }\end{array}$ \\
\hline Avaliador 1 & 20 & $20.48 \pm 4.00$ & $21.57 \pm 3.00$ \\
Avaliador 2 & 20 & $20.26 \pm 4.43$ & $20.57 \pm 4.55$ \\
Avaliador 3 & 20 & $19.57 \pm 3.53$ & $20.44 \pm 2.49$ \\
Valor de P & & 0,20 & 0,64 \\
\hline
\end{tabular}

Acredita-se que a menor variação obtida nos resultados do presente trabalho se dê em razão das facilidades proporcionadas pelo software. Enquanto no cálculo manual descrito por Slocum e Devine (1983), são confeccionadas três linhas, sendo a primeira no eixo mecânico da tíbia, a segunda na superfície do platô e a terceira perpendicular ao eixo mecânico no ponto de intersecção das duas linhas, finalizando com o valor do ângulo obtido por meio do uso do transferidor, no cálculo por software o valor do ângulo preciso é fornecido automaticamente após o traçado das duas primeiras linhas, o que reduz a chance de erro causada pelo uso do transferidor utilizado no cálculo manual.

As imagens digitais podem ser trabalhadas quanto à ampliação, às correções de técnicas e à densidade da imagem, o que auxilia na localização dos pontos de referência (Serwa et al., 2009), sendo outro benefício da imagem digital em relação à impressa. Entretanto, no presente estudo, as imagens digitais não foram trabalhadas durante o cálculo do ângulo do platô 
tibial, para que os métodos fossem comparados sem influência dessas variáveis.

No cálculo manual, o traçado das linhas nos pontos de referência, a espessura da caneta utilizada e o uso do transferidor podem levar a pequenas variações que, no valor final, podem levar a grandes diferenças. No presente estudo, o material utilizado pelos avaliadores foi idêntico (caneta, régua plástica de 30 centímetros e transferidor plástico).

Não foram observadas diferenças quando comparados os ângulos do platô do perfil direito e do esquerdo dos cães em ambos os métodos, o que demonstra a simetria em cães saudáveis.

\section{CONCLUSÃO}

Conclui-se que há diferença dos valores obtidos por meio dos métodos convencional e digital no cálculo do ângulo do platô tibial. O método digital apresenta superioridade em relação à facilidade no cálculo e à menor variação de valores e cães saudáveis apresentam simetria no valor do ângulo do platô tibial dos membros pélvicos.

\section{REFERÊNCIAS}

BUQUERA, L.E.C.; PADILHA FILHO, J.G.; CANOLA, J.C. Ruptura do ligamento cruzado cranial em cães - revisão de literatura. Arq. Cienc. Vet. Zool., v.7, p.43-47, 2004.

FETTIG, A.A.; RAND, W.M.; SATO, A.F. et al. Observer variability of tibial plateau slope measurement in 40 dogs with cranial cruciate ligament deficient stifle joints. Vet. Surg., v.32, p.471-478, 2003.

GRIFFON, D.J. A review of the pathogenesis of canine cranial cruciate ligament disease as a basis for future preventive strategies. Vet. Surg., v.39, p.399-409, 2010.
HARASEN, G. Diagnosing rupture of the cranial cruciate ligament. Can. Vet. J., v.43, p.475-476, 2002.

JOHNSON, J.M.; JOHNSON, A.L. Cranial cruciate ligament rupture: pathogenesis, diagnosis, and postoperative rehabilitation. Vet. Clin. N. Am. Small Anim. Pract., v.23, p.717733, 1993.

LAZAR, T.P. et al. Long-team radiographic comparison of tibial plateau leveling osteotomy verus extracapsular stabilization for cranial cruciate ligament rupture in the dog. Vet. Surg., v.34, p.133-141, 2005.

MORRIS, E.; LIPOWITZ, A.J. Comparison of tibial plateau angles in dogs with and without cranial cruciate ligament injuries. J. Am. Vet. Med. Assoc., v.218, p.363-366, 2001.

REIF, U.; DEJARDIN, L.M.; PROBST, C.W. et al. Influence of limb positioning and measurement method on the magnitude of the tibial plateau angle. Vet. Surg., v.33, p.368-375, 2004.

SERWA, D.; LORINSON, K.; LORINSON, D. et al. Comparison of conventional and digital measurements of tibial plateau angle in dogs. $J$. Am. Vet. Med. Assoc., v.234, p.622-624, 2009.

SLOCUM, B.; DEVINE, T. Cranial tibial thrust: a primary force in the canine stifle. J. Am. Vet. Med. Assoc., v.183, p.456-459, 1983.

WILKE, V.L.; CONZEMIUS, M.G.; BESANCON, M.F. et al. Comparison of tibial plateau angle between clinically normal Greyhounds and Labrador Retrievers with and without rupture of the cranial cruciate ligament. J. Am. Vet. Med. Assoc., v.221, p.1426-1429, 2002. 